\title{
Menstrual Suppression Using Oral Contraceptives by Female Hajj Pilgrims
}

\author{
Wealeed Dandehbor ${ }^{1}$, Majid Kazemi ${ }^{2}$, Mohammad Hossein Salehi Shahrbabaki ${ }^{3}$, Zohre Ghorashi ${ }^{4}$, \\ Rezvan Sadrmohammadi ${ }^{5}$, Bonnie Bozorg ${ }^{6}$, Reza Bidaki ${ }^{7,8}$ \\ 'Deputy of Medical Centre Hajj and Plgrimage of Iranian Red Crescent Society, Tehran, Iran \\ ${ }^{2}$ Department Medical Surgical Nursing, Faculty of Nursing and Midwifery, Rafsanjan University of Medical Sci- \\ ences, Rafsanjan, Iran \\ ${ }^{3}$ Rafsanjan University of Medical Sciences, Rafsanjan, Iran \\ ${ }^{4}$ Department of Midwifery, Rafsanjan University of Medical Sciences, Rafsanjan, Iran \\ ${ }^{5}$ Department of Clinical Psychology, Kar Higher Education Institute of Rafsanjan, Rafsanjan, Iran \\ ${ }^{6}$ Tehran University of Medical Sciences, Tehran, Iran \\ ${ }^{7}$ Research center of Addiction and Behavioral sciences, Shahid Sadoughi University of Medical Sciences, Yazd, Iran \\ ${ }^{8}$ Diabetes Research Center, Shahid Sadoughi University of Medical Science, Yazd, Iran
}

Corresponding Author: Majid Kazemi, Ph.D., Associate Professor of Surgical Nursing, School of Nursing, Rafsanjan University of Medical Sciences, Rafsanjan, Iran. Tel: 03434255900; Fax: 03434258497; Email: maj_kaz@yahoo.com

Received September 3, 2016; Accepted October 20, 2016; Online Published December 5, 2016

\begin{abstract}
Introduction: The pilgrimage to Ka'beh (God's house in Mecca) is a special opportunity in the life of most Muslims. Female pilgrims try to postpone menstruation during the pilgrimage by taking hormonal medicines. However, women are seen in this disorder. This paper aimed to determine the frequency of menstrual disorders and related factors among female pilgrims of Umrah Mufradah in 2012.

Methods: This study was a descriptive type, the population of which comprised 400 female Iranian pilgrims aged 15-50 years who were selected through random cluster sampling from Umrah caravans. Data was collected with a questionnaire developed by the researchers that contained questions about menstrual disorders. The questionnaire was distributed among participants at their hotels three days prior to their return to Iran.

Results: Among all the participants, $98.7 \%$ of them reported taking pills to suppress menstruation. Of this group, $74.7 \%$ successfully prevented menstruation, $26 \%$ reported spotting, and $11.6 \%$ reported menstruation. There was no significant difference between consumption of medicine and factors such as marital status, city, and educational background of the individuals. Participants reported that spotting and menstruation caused them to experience stress and anxiety when performing Umrah rituals.

Conclusion: Experiencing menstrual disorders during pilgrimage can be stressful for pilgrims. To reduce such problems, counseling sessions on menstruation postponement as part of the justification classes before departing for Hajj and compiling an equal and coordinated nationwide protocol seem necessary.
\end{abstract}

Keywords: Menstrual, Oral contraceptives, Hajj pilgrims

Citation: Dandehbor W, Kazemi M, Salehi Shahrbabaki MH, et al. Menstrual suppression using oral contraceptives by female Hajj pilgrims. Int J Travel Med Glob Health. 2016;4(4):111-114. doi:10.21859/ijtmgh-040405.

\section{Introduction}

The Hajj ceremony is the most important life event for any Muslim. ${ }^{1}$ For the majority of pilgrims, it is a long-desired journey that may happen only once in a lifetime. Annually, millions of Muslims from various countries gather in the holy cities of Mecca and Medina in the Kingdom of Saudi Arabia to perform their religious rituals..$^{2-4}$

According to religious instructions, purity or cleanness is a condition for entering Masjed Al'Haram (the respected mosque) or other mosques. Women are not permitted to enter mosques or say their prayers while menstruating. ${ }^{2}$

To take the greatest advantage of their pilgrimage, many women of reproductive age try to prevent menstruation during the pilgrimage ${ }^{3}$ by taking oral hormonal medicines. The first case of taking medicine to postpone menstruation dates back to $1977 .{ }^{3}$ Today, this method is used to decrease the number of menstruation cycles. Similar utilization of hormonal medicines is common among Jewish females. ${ }^{4}$

Definitely, delaying menstruation by taking hormonal medicines intermittently and for short periods to remove

Copyright (C) 2016 The International Journal of Travel Medicine and Global Health. This is an open-access article distributed under the terms of the Creative Commons Attribution License (http://creativecommons.org/licenses/by/4.0), which permits unrestricted use, distribution, and reproduction in any medium, provided the original work is properly cited. 
impediments to religion, is regarded as a special case and has its own side effects and problems. ${ }^{5}$ Usually, women who are willing to use this method of prevention have not taken these medicines before and are more likely to experience more side effects. The emotional effects of contraceptive pills can be worse than their physical effects. ${ }^{6}$ The inefficiency of these medicines in delaying menstruation appears mainly in the form of spotting during consumption and can endanger the main philosophy of consumption, to remove impediments to religion, and cause stress and other problems for the consumers. Some studies have investigated the consumption of these medicines, and spotting while on the medicine has been reported as a main complaint of pilgrims., ${ }^{2,7}$ Some women who use contraceptive pills during their pilgrimage experience side effects. The most important and most commonly occurring side effect of the contraceptive pill is spotting, which can lead to anxiety and depression.

Based on informal reports of menstrual disorders among pilgrims in Mecca, the current study aimed to determine the prevalence of menstrual disorders and related factors in Mufradah pilgrims in 2012.

\section{Methods}

This descriptive study considered 400 female Iranian pilgrims aged 15-50 years who did not have an intrauterine device (IUD). The participants were selected through random cluster sampling from Umrah caravans. The size of the population was determined to be 350 people based on similar studies, but for the sake of higher reliability 400 participants were studied. The exclusion criteria included women who were reluctant or unwilling to participate in the study. The instrument used in this study was a two-part questionnaire developed by the researchers and based on pilgrims' complaints and questionnaires from prior studies. The first section contained questions regarding personal characteristics, and the second section involved questions about methods of menstruation prevention, symptoms of menstrual disorders, spotting, and treatment precautions. The questionnaire included 3 openended questions about the side effects of the medicine, the emotional effects of the resultant disorder, and the impact the resultant disorder had on the performance of Umrah rituals.

The questionnaire consisted of 23 questions, 5 regarding demographic data, 15 regarding menstruation and medicine consumption for menstrual suppression, and 3 open-answer questions which addressed side effects and problems.

Content validity of the questionnaire was checked through the opinions of 3 gynecologists and 3 faculty members of the Rafsanjan University of Medical Sciences. The reliability of the questionnaire was 0.84 . The questionnaire was distributed among the participants at their hotels 3 days before their return to Iran. Random cluster sampling was used to randomly select hotels and the Iranian caravans staying in them. Women from each caravan volunteered to distribute and collect the questionnaires. The collected data was inserted into SPSS version 18 software and analyzed using parametric and nonparametric tests such as the chi-square test.

\section{Results}

Four hundred female Umrah pilgrims participated in the study. The average age of the participants was $34.4 \pm 1.33 ; 19.2$
$\%$ of them were single, and $80.8 \%$ were married. The results indicated that $53.2 \%$ were housewives, $44.7 \%$ had a bachelor's degree or higher, and $2 \%$ of them were illiterate.

Results indicated that $98.7 \%$ of participants reported taking birth control pills to suppress menstruation, and $74.7 \%$ of them reported successfully preventing it. The average number of pills used per day was $1.13 \pm 0.45$. The average start time for taking medicine before menstruation was $10.37 \pm 9.7 ; 51.5 \%$ of participants received consulted regarding the pills, and $19.9 \%$ of them had no consultation at all before the pilgrimage. Consultation was received by $42.4 \%$ of participants one week, $14.7 \%$ two weeks, $6.8 \%$ three weeks, and $36.7 \%$ more than 21 days prior to departing for Hajj. The average time before departure for counseling was $22.23 \pm 23.95$ days (Table 1 ).

Despite taking medicine to postpone menstruation, $26 \%$ of the participants reported spotting. Spotting was more frequent in participants aged 25-45 years than participants under the age of 25 years or over 45 years. Statistically, the differences were significant at $P=0.03$ (Table 2). The average start time for spotting was on the eleventh day of the menstrual cycle $(11 \pm 11.88)$. Also, $11.6 \%$ of the individuals taking medicine

Table 1. Sample Frequency in Terms of Job, Educational Background, Prevention Method, Counseling and the Time of it

\begin{tabular}{|c|c|c|}
\hline Variables & No. & Percent \\
\hline \multicolumn{3}{|l|}{ Job } \\
\hline Teacher & 56 & 14.2 \\
\hline Housewife & 210 & 53.2 \\
\hline Student & 74 & 18.7 \\
\hline Employee & 55 & 13.9 \\
\hline Total & 395 & 100 \\
\hline \multicolumn{3}{|l|}{ Educational background } \\
\hline Illiterate & 8 & 2 \\
\hline Lower than diploma & 76 & 18.7 \\
\hline Diploma & 141 & 34.6 \\
\hline Bachelor's degree & 151 & 37.1 \\
\hline Higher than a bachelor's degree & 31 & 7.8 \\
\hline Total & 407 & 100 \\
\hline \multicolumn{3}{|l|}{ Prevention method } \\
\hline No prevention & 106 & 25.3 \\
\hline Pill & 302 & 72.1 \\
\hline Injection & 5 & 1.2 \\
\hline Injection and pill & 6 & 1.4 \\
\hline Total & 419 & 100 \\
\hline \multicolumn{3}{|l|}{ Counseling } \\
\hline Doctor & 168 & 51.5 \\
\hline Midwife & 60 & 18.4 \\
\hline Friends & 33 & 10.1 \\
\hline No counseling & 65 & 19.9 \\
\hline Total & 326 & 100 \\
\hline \multicolumn{3}{|l|}{ Time of counseling } \\
\hline One week before departure & 75 & 42.4 \\
\hline One to two weeks before departure & 26 & 14.7 \\
\hline Two to four weeks before departure & 16 & 9.1 \\
\hline One to two months before departure & 56 & 31.6 \\
\hline More than two months & 4 & 2.2 \\
\hline Total & 177 & 100 \\
\hline
\end{tabular}


Table 2. Spotting Frequency by Age Group

\begin{tabular}{|c|c|c|c|c|c|c|}
\hline \multirow{2}{*}{ Age } & \multicolumn{2}{|c|}{ Spotting } & \multicolumn{2}{|c|}{ No Spotting } & \multicolumn{2}{|c|}{ Total } \\
\hline & No. & Percent & No. & Percent & Percent & No. \\
\hline Less than 25 & 16 & 18.8 & 69 & 81.2 & 100 & 85 \\
\hline $25-45$ & 61 & 33 & 124 & 67 & 100 & 185 \\
\hline More than 45 & 6 & 14 & 37 & 86 & 100 & 43 \\
\hline
\end{tabular}

Table 3. Comparison of Delay in Menstruation and Educational Background

\begin{tabular}{|c|c|c|c|c|c|c|}
\hline \multirow{2}{*}{ Educational Background } & \multicolumn{2}{|c|}{ Menstruation Delay } & \multicolumn{2}{|c|}{ No Menstruation Delay } & \multicolumn{2}{|c|}{ Total } \\
\hline & No. & Percent & No. & Percent & No. & Percent \\
\hline Illiterate & 5 & 62.5 & 3 & 27.5 & 8 & 100 \\
\hline Diploma & 107 & 75.9 & 34 & 24.1 & 141 & 100 \\
\hline Bachelor's degree & 122 & 80.8 & 29 & 19.2 & 151 & 100 \\
\hline Higher than a bachelor's degree & 22 & 70.9 & 9 & 29.1 & 31 & 100 \\
\hline
\end{tabular}

to postpone menstruation reported menstruation. To obviate spotting, $74.6 \%$ had referred to a doctor; of those individuals, $61.6 \%$ reported their spotting was obviated and $27.9 \%$ reported increasing the dose of the medicine to obviate this problem.

Among the participants, $41 \%$ of those who consumed medicine experienced at least one medicinal side effect, mostly sickness and vomiting.

There was no significant difference between menstruation delay and individuals' educational background; however, the rate of medicine consumption was higher among persons holding a college degree (Table 3 ). There was no significant difference at $P>0.05$ between menstruation delay and factors such as marital status and the dispatch city in Iran. There was a significant difference between the consumption of medicine and the age category; fewer participants over the age of 45 years took medicine than the others.

Qualitative analysis of the open-ended questions showed that spotting and menstruation were reported as stressful for participants in performing Umrah rituals. Some participants reported menstruation as causing feelings of their visit to the Ka'beh being rejected by God and expressed experiencing great stress and anxiety.

\section{Discussion}

Results of the current study indicated that $74.7 \%$ of the female pilgrims successfully prevented menstruation during their pilgrimage to the Ka'beh (God's house in Mecca), and most of them reported using contraceptive pills to prevent it. Currently, the long-term use of contraceptive pills to decrease the occurrence of menstruation has been of great interest to researchers and women of reproductive age. Large numbers of women from all over the world prefer to take contraceptive pills to prevent menstruation for many days. ${ }^{5,8}$

Despite taking pills, $26 \%$ of the pilgrims participating in the current study experienced spotting and $11.6 \%$ menstruated. The average start time for spotting was on the eleventh day of the menstrual cycle. Spotting is a common side effect of contraceptive pills, even in the common 21-day method of using them. ${ }^{9}$ Most cases of spotting in this study can be attributed to the short interval between starting medication and spotting. Since contraceptive pills have a low amount of estrogen in them, a low dose of conjugated estrogen is easily used to alleviate the problem of spotting. Furthermore, with the low dose of conjugated estrogen, the user is not disposed to the side effects of the higher hormone dosage.

In a study by Sulak et al on 318 patients, they found that the pills decrease the symptoms of PMS in 13\%, hypermenorrhea $19 \%$ and headache $35 \% .{ }^{10}$ Other studies which applied extended and continuous regimens for preventing menstruation reported a high and variable prevalence of spotting and bleeding; however, these unwanted side effects were eliminated after a few cycles. A high prevalence of side effects was reported in women who used contraceptive pills for the first time.

Ghorashi et al reported the occurrence rates of spotting and menstrual bleeding in Hajj pilgrims in 2000 as $13.6 \%$ and $2.1 \%$, respectively. ${ }^{11}$ The low occurrence of these side effects in the study of Ghorashi et al compared with the results of the present study can be attributed to the high dose of the pills taken by participants. In the study of Ghorashi et al, about $20 \%$ of the individuals took HD pills from the beginning, and the others took two LD pills simultaneously.

In the present study, more than half of the individuals aged over 45 years had used hormonal medicines to postpone menstruation. An increase in the age of the user increased the side effects of the contraceptive pills. ${ }^{8}$ Moreover, most of the middle-aged women in this study suffered from diseases like diabetes or hypertension, both of which can endanger the consumption of contraceptive pills. Therefore, special training and care for postponing menstruation, such as the use of non-estrogenic pills, should be allocated to this group.

Statistically, there was no significant difference between the start time for taking pills and the occurrence of spotting. Therefore, those women who had a delayed start in taking pills during their menstrual cycle did not report the occurrence of spotting any more than those who started taking the pill on the fifth day of their menstrual cycle. Furthermore, Ghorashi et al found no significant difference between these two variables. It can be concluded that the delay in starting medicine consumption did not create additional problems for the users. This will be helpful for those who tend to decrease the consumption of the pill for different reasons. Therefore, 
decreasing the number of pills can help decrease their side effects. $^{7}$

About $20 \%$ of the participants in this study who had used menstruation postponement methods had received no counseling on the use of medicine before starting consumption. From those who reported receiving counseling, more than $60 \%$ reported receiving counseling a short time (three weeks or less) before the pilgrimage. It should be noted that unadvised use of contraceptive pills among those who are at a high risk can be dangerous or even deadly. Furthermore, the unadvised use of contraceptive pills can lead to inappropriate consumption, the possibility of increased side effects, and a lack of necessary instruction on how to cope with side effects such as spotting. Therefore, it is suggested that counseling be taken into account in justification classes.

It is recommended that justification classes be held duly and more than one month before the pilgrimage to allow women to make better decisions about how to postpone menstruation.

The most common side effect of taking contraceptive pills noted in this study was vomiting, which is a prevalent side effect of contraceptive pills. ${ }^{12}$ Because of the short period of consumption and the specificity of the occasion, it is essential to advise pilgrims on possible side effects of contraceptive pills and the necessity of having them during the pilgrimage. ${ }^{13}$ Since the Hajj pilgrimage is an important and sometimes exclusive event in the life of most pilgrims, the experience of menstrual disorders during pilgrimage can be stressful, disappointing, and problematic for them. ${ }^{14}$ To reduce such problems, holding counseling sessions on menstruation postponement as a part of the justification classes before departure for Hajj and compiling an equal and coordinated nationwide protocol seem necessary. ${ }^{15}$

\section{Conclusion}

The side effects of taking contraceptive pills for menstrual suppression and their failures are considerable and cause pilgrims to feel much stress and inconvenience. It is suggested that this traditional approach be replaced with progestin-only pills, such as megestrol.

\section{Authors' Contributions}

All authors contributed significantly toward this study.

\section{Conflict of Interest Disclosures}

None declared.

\section{Ethical Approval}

Not applicable.

\section{Funding/Support}

There was no funding support for this research.

\section{Acknowledgements}

The authors would like to thank all the female pilgrims who graciously completed the questionnaire in the short time of their pilgrimage.

\section{References}

1. Al-Ghamdi AS, Kabbash IA. Awareness of healthcare workers
Research Highlights

\section{What Is Already Known?}

It is already known the side effects of taking contraceptive pills for menstrual suppression and their failures are considerable and cause pilgrims to feel much stress and inconvenience. This review elaborates on the effects of contraceptive pills on travelers.

\section{What This Study Adds?}

The results of the current study suggest that the traditional approach of using contraceptive pills to suppress menstruation should be replaced with the consumption of progestin-only pills, such as megestrol pills. It is essential that pilgrims be advised about the possible side effects of the pills and the necessity of having them during the pilgrimage.

regarding preventive measures of communicable diseases among Hajj pilgrims at the entry point in Western Saudi Arabia. Saudi Med J. 2011;32(11):1161-1167.

2. Al-Ghamdi SM, Akbar HO, Qari YA, Fathaldin OA, Al-Rashed RS. Pattern of admission to hospitals during muslim pilgrimage (Hajj). Saudi Med J. 2003;24(10):1073-1076.

3. Azarpazhooh MR, Rafi S, Etemadi MM, Khadem N, Fazlinejad A. The relation between short-term oral contraceptive consumption and cerebrovascular, cardiovascular disorders in Iranian women attending Hajj. Saudi Med J. 2008;29(7):1024-1027.

4. Bakhsh AR, Sindy AI, Baljoon MJ, Dhafar KO, Gazzaz ZJ, Baig $M$, et al. Diseases pattern among patients attending Holy Mosque (Haram) Medical Centers during Hajj 1434 (2013). Saudi Med J. 2015;36(8):962-966. doi:10.15537/smj.2015.8.12120.

5. Berek JS. Novak's Text Book of Gynecology. 13th ed. Baltimore: Williams \& Wlkins; 2002:250-261.

6. Burkman RT. Oral contraceptives current status. Clin Obstet Gynecol. 2001;44(1):62-72. doi:10.1097/00003081-20010300000010.

7. Madani TA, Ghabrah TM, Al-Hedaithy MA, et al. Causes of hospitalization of pilgrims in the Hajj season of the Islamic year 1423 (2003). Ann Saudi Med. 2006;26(5):346-51.

8. Mansour D, Experiences with Yasmin; the acceptability of a novel oral contraceptive and its effect on well being. Eur J Contracept Reprod Health Care. 2002; 7 Suppl 13:35-41.

9. Elachola $\mathrm{H}$, Assiri A, Turkestani AH, et al. Advancing the global health security agenda in light of the 2015 annual Hajj pilgrimage. Int J Infec Dis. 2015. doi:10.1016/j.ijid.2015.10.003.

10. Sulak PJ, Kuehl TJ, Ortiz M, Shull BL. Acceptance of altering the standard 21-day/7day oral contraceptive regimen to delay menses and reduce hormone withdrawal symptoms. Am J Obstet Gynecol. 2002;186(6):1142-1149.

11. Ghorashi Z, Taleghani F, Shafiee M. Failure and side effects of contraceptive pills used for postponement of menstrual bleeding in Hajj Pilgrims of Kerman (2000). JSSU. 2005;12(4):65-70.

12. Riaz SA, Ahmadi M, Mortazavi SM. Predictors of anxiety and depression among women with vaginal bleeding referred to gynecology clinic during hajj 2011. Iran Red Crescent Med J. 2014;16(11):e12855. doi:10.5812/ircmj.12855.

13. Masood K, Gazzaz ZJ, Ismail K, Dhafar KO, Kamal A. Pattern of psychiatry morbidity during Hajj period at Al-Noor specialist hospital. Int J Psychiatry Med. 2007;37(2):163-172.

14. Mohammed-Durosinlorun A, Raji HO, Hussain NA, Badmus SA, Ijaiya MA. Menstrual suppression among female Nigerian pilgrims during Hajj. J Fam Plann Reprod Health Care. 2012;38(4):270271. doi:10.1136/jfprhc-2012-100401.

15. Tin SS, Wiwanitkit V. Hajj pilgrimage surveillance in pilgrims from African countries. Trop Med Int Health. 2015;20(2):E11. doi:10.1111/tmi.12413. 